\title{
Cross sections for electron impact excitation of the low-lying electron states of $\mathrm{CO}_{2}$
}

\author{
Lee $\mathrm{Mu}$-Tao ${ }^{\dagger}$ and Vincent McKoy \\ A A Noyes Laboratory of Chemical Physics ł, California Institute of Technology, Pasadena, \\ California 91125 , USA
}

Received 19 May 1982, in final form 14 October 1982

\begin{abstract}
Distorted-wave cross sections for the excitation of eight low-lying excited states $\left({ }^{1,3} \Sigma_{\mathrm{u}}^{+},{ }^{1,3} \Pi_{\mathrm{g}},{ }^{1,3} \Pi_{\mathrm{u}}\right.$ and $\left.{ }^{1,3} \Delta_{\mathrm{u}}\right)$ of $\mathrm{CO}_{2}$ by electrons in the 25 to $60 \mathrm{eV}$ energy range are presented. We report both differential and integral cross sections.
\end{abstract}

\section{Introduction}

Knowledge of the electron impact excitation cross sections of the low-lying electronic states of $\mathrm{CO}_{2}$ at low and intermediate energies is of great importance in many processes, e.g., the dissociation and recombination of $\mathrm{CO}_{2}$ which occurs in planetary atmospheres and in the $\mathrm{CO}_{2}$ laser (Land 1978, Strickland and Green 1969). Despite the wealth of experimental studies in both optical (Tanaka et al 1960, Inn et al 1953, Price and Simpson 1938, Rabalais et al 1971) and electron impact (Lassettre and Shiloff 1965, Meyer and Lassettre 1965, Lassettre et al 1968, Hall et al 1973) spectroscopy, we are unaware of any studies of the electronic excitation cross section of $\mathrm{CO}_{2}$ in the low and intermediate impact energy range. Generalised oscillator strengths for some singlet excited states have been reported (McCurdy and McKoy 1974). Such Born-type approximations are known to be unreliable at low and intermediate impact energies.

In view of the importance of the $\mathrm{CO}_{2}$ molecule in several applications, we have calculated the differential and integral cross sections for excitation of eight low-lying $\left({ }^{1,3} \Sigma_{\mathrm{u}}^{+},{ }^{1,3} \Pi_{\mathrm{g}},{ }^{1,3} \Pi_{\mathrm{u}}\right.$ and $\left.{ }^{1,3} \Delta_{\mathrm{u}}\right)$ excited states of $\mathrm{CO}_{2}$ by electron impact for impact energies in the 25 to $60 \mathrm{eV}$ range by a distorted-wave method. We have recently applied this distorted-wave method to study the electronic excitation processes in $\mathrm{H}_{2}$ (Lee et al 1982), $\mathrm{N}_{2}$ (Lee and McKoy 1982a) and CO (Lee and McKoy 1982b). The results of these applications of the DW approach to systems such as $\mathrm{H}_{2}$ and $\mathrm{N}_{2}$, for which some experimental data are available, allow us to assess the reliability of the method. These results showed that, while the DW method generally reproduced the shape of the measured differential cross sections quite well, the integral cross sections could be in error by up to a factor of two or three in some cases. In view of the very limited experimental data available for most systems, and the significant difficulties involved in applying more sophisticated theoretical approaches, these results help to establish the utility of the DW approach to electronic excitation cross sections. We

\footnotetext{
$\uparrow$ Permanent address: Departamento de Quimica, Universidade Federal de São Carlos, 13560, S P Brasil.
} $\ddagger$ Contribution No 6612 . 
hence expect that these DW differential and integral cross sections could be useful both in the interpretation of many processes that occur in plasmas containing $\mathrm{CO}_{2}$ and in the analysis of experimental data.

The theory used in these calculations has already been presented (Fliflet and McKoy 1980, Lee et al 1982). In $\$ 2$ we will discuss some details of the calculations and the results will be presented.

\section{Calculations}

In this Dw method, the elastic continuum wavefunctions are obtained in the staticexchange potential field of the ground state of $\mathrm{CO}_{2}$ by the iterative Schwinger variational method (Lucchese et al 1980). The SCF wavefunction of the ground state at the equilibrium distance of 2.1944 au $\left(R_{\mathrm{C}-\mathrm{O}}\right)$ was obtained using the standard $[3 \mathrm{~s}, 2 \mathrm{p}]$ basis of contracted Gaussian functions constructed from the [9s, 5p] set of Dunning (1970), augmented by a set of $(1 \mathrm{~s}, 1 \mathrm{p}, 1 \mathrm{~d})$ functions on the nuclei $\left(\alpha_{\mathrm{C}}=0.036\right.$, 0.030 and $0.75 ; \alpha_{O}=0.048,0.04$ and 0.85 respectively). This basis set gives a total energy of -187.67634 au and a quadrupole moment of -4.106 au.

The details of the iterative Schwinger variational method have already been given by Lucchese et al (1980). We obtained the continuum wavefunctions for partial waves with $l \leqslant 10$ and $m \leqslant 2$. In these calculations we retained partial waves up to $l=108$ in the direct potential and up to 40 in the exchange potential. The maximum partial wave retained in the expansion of all other functions in this calculation was $l=59$. The initial basis sets used in the calculation for the continuum wavefunctions of $\sigma_{\mathrm{g}}$, $\sigma_{\mathrm{u}}, \pi_{\mathrm{g}}$ and $\pi_{\mathrm{u}}$ symmetries have been presented elsewhere (Lucchese and McKoy 1982). The basis sets for the continuum wavefunctions of $\delta_{\mathrm{g}}$ and $\delta_{\mathrm{u}}$ symmetries were chosen in a similar way. Only one iteration was needed in the Schwinger iterative procedure. Calculations were carried out for incident energies in the $25-60 \mathrm{eV}$ energy range. To determine the energy of the scattered electron for a given impact energy, we used the experimental vertical excitation energies for the excited states (HubinFranskin and Collins 1971, Rabalais et al 1971, Lassettre and Shiloff 1965) except for the ${ }^{3} \Pi_{g}$ and ${ }^{3} \Pi_{u}$ states. For these two excited states, the calculated vertical excitation energies (McCurdy and McKoy 1974) were used.

For the excited states of the target, we used configuration interaction wavefunctions. These wavefunctions included all single excitations from the occupied $3 \sigma_{\mathrm{g}}, 4 \sigma_{\mathrm{u}}, 1 \pi_{\mathrm{g}}$ and $1 \pi_{u}$ orbitals to the virtual orbitals of the appropriate symmetry. These wavefunctions contained 32 configurations for the ${ }^{1,3} \Sigma_{\mathrm{u}}^{+},{ }^{1,3} \Pi_{\mathrm{g}}$ and ${ }^{1,3} \Pi_{\mathrm{u}}$ states and 20 configurations for the ${ }^{1,3} \Delta_{\mathrm{u}}$ states.

\section{Results}

In tables 1 to 8 , we present our DW differential and total cross sections for excitation of these eight low-lying states, along with the Born total cross sections for the corresponding singlet-type transitions, obtained with the same target wavefunctions for impact energies of 30,40 and $50 \mathrm{eV}$. As expected, the agreement between the DW and Born cross sections improves with increasing impact energies.

In figures 1-6, we present the differential cross sections for excitation of these eight states at two impact energies $(25$ and $60 \mathrm{eV})$. For the singlet-singlet type 
Table 1. Calculated DW differential and integral cross sections (in $10^{-16} \mathrm{~cm}^{2} \mathrm{sr}^{-1}$ and $10^{-16} \mathrm{~cm}^{2}$ ) and Born integral cross sections for excitation of the ${ }^{1} \Pi_{\mathrm{g}}$ state.

\begin{tabular}{llll}
\hline & \multicolumn{3}{c}{ Energy $(\mathrm{eV})$} \\
\cline { 2 - 3 } Angle (deg) & 30 & 40 & 50 \\
\hline 10 & $8.88(-2)$ & $9.35(-2)$ & $9.46(-2)$ \\
20 & $5.46(-2)$ & $4.54(-2)$ & $3.58(-2)$ \\
30 & $2.33(-2)$ & $1.25(-2)$ & $6.47(-3)$ \\
40 & $6.94(-3)$ & $2.26(-3)$ & $1.64(-3)$ \\
50 & $1.80(-3)$ & $1.95(-3)$ & $2.91(-3)$ \\
60 & $1.56(-3)$ & $3.04(-3)$ & $3.53(-3)$ \\
70 & $2.32(-3)$ & $3.21(-3)$ & $3.15(-3)$ \\
80 & $2.69(-3)$ & $2.70(-3)$ & $2.35(-3)$ \\
90 & $2.57(-3)$ & $2.00(-3)$ & $1.57(-3)$ \\
100 & $2.21(-3)$ & $1.36(-3)$ & $8.72(-4)$ \\
110 & $1.82(-3)$ & $8.58(-4)$ & $4.29(-4)$ \\
120 & $1.46(-3)$ & $5.28(-4)$ & $2.31(-4)$ \\
130 & $1.20(-3)$ & $3.88(-4)$ & $1.91(-4)$ \\
140 & $1.08(-3)$ & $3.94(-4)$ & $2.49(-4)$ \\
150 & $1.16(-3)$ & $4.37(-4)$ & $3.59(-4)$ \\
160 & $1.37(-3)$ & $4.31(-4)$ & $4.29(-4)$ \\
170 & $1.59(-3)$ & $3.79(-4)$ & $4.04(-4)$ \\
180 & $1.68(-3)$ & $3.49(-4)$ & $3.70(-4)$ \\
Total Dw & $7.68(-2)$ & $6.23(-2)$ & $5.45(-2)$ \\
Born & $5.33(-2)$ & $4.33(-2)$ & $3.61(-2)$ \\
\hline & & &
\end{tabular}

Table 2. Calculated DW differential and integral cross sections (in $10^{-16} \mathrm{~cm} \mathrm{sr}^{-1}$ and $10^{-16} \mathrm{~cm}^{2}$ ) and Born integral cross sections for excitation of the ${ }^{1} \Pi_{\mathrm{u}}$ state.

\begin{tabular}{llll}
\hline & \multicolumn{3}{c}{ Energy (eV) } \\
\cline { 2 - 4 } Angle (deg) & 30 & 40 & 50 \\
\hline 10 & $6.45(-1)$ & $6.87(-1)$ & $6.36(-1)$ \\
20 & $2.20(-1)$ & $1.58(-1)$ & $1.10(-1)$ \\
30 & $7.08(-2)$ & $3.87(-2)$ & $2.31(-2)$ \\
40 & $2.26(-2)$ & $1.06(-2)$ & $6.20(-3)$ \\
50 & $7.40(-3)$ & $3.94(-3)$ & $2.89(-3)$ \\
60 & $3.07(-3)$ & $2.42(-3)$ & $1.94(-3)$ \\
70 & $2.00(-3)$ & $1.77(-3)$ & $1.34(-3)$ \\
80 & $1.66(-3)$ & $1.28(-3)$ & $9.59(-4)$ \\
90 & $1.45(-3)$ & $1.01(-3)$ & $7.72(-4)$ \\
100 & $1.30(-3)$ & $8.93(-4)$ & $7.14(-4)$ \\
110 & $1.17(-3)$ & $8.66(-4)$ & $7.07(-4)$ \\
120 & $1.08(-3)$ & $8.97(-4)$ & $6.88(-4)$ \\
130 & $1.03(-3)$ & $9.43(-4)$ & $6.65(-4)$ \\
140 & $1.06(-3)$ & $9.38(-4)$ & $6.76(-4)$ \\
150 & $1.16(-3)$ & $8.50(-4)$ & $7.26(-4)$ \\
160 & $1.30(-3)$ & $7.10(-4)$ & $8.06(-4)$ \\
170 & $1.42(-3)$ & $5.83(-4)$ & $8.75(-4)$ \\
180 & $1.47(-3)$ & $5.32(-4)$ & $9.06(-4)$ \\
Total DW & $3.04(-1)$ & $2.63(-1)$ & $2.12(-1)$ \\
Born & $1.51(-1)$ & $1.46(-1)$ & $1.31(-1)$ \\
\hline
\end{tabular}


Table 3. Calculated DW differential and integral cross sections (in $10^{-16} \mathrm{~cm}^{2} \mathrm{sr}^{-1}$ and $10^{-16} \mathrm{~cm}^{2}$ ) and Born integral cross sections for excitation of the ${ }^{1} \Sigma_{u}^{+}$states.

\begin{tabular}{llll}
\hline & \multicolumn{3}{c}{ Energy (eV) } \\
\cline { 2 - 4 } Angle (deg) & 30 & 40 & 50 \\
\hline 10 & $7.70(-2)$ & $9.30(-2)$ & $8.98(-2)$ \\
20 & $1.01(-2)$ & $6.60(-3)$ & $4.38(-3)$ \\
30 & $1.81(-3)$ & $2.93(-3)$ & $3.61(-3)$ \\
40 & $2.27(-3)$ & $2.81(-3)$ & $2.18(-3)$ \\
50 & $1.55(-3)$ & $1.26(-3)$ & $6.86(-4)$ \\
60 & $6.49(-4)$ & $4.36(-4)$ & $3.08(-4)$ \\
70 & $3.43(-4)$ & $2.77(-4)$ & $2.30(-4)$ \\
80 & $3.84(-4)$ & $2.15(-4)$ & $1.61(-4)$ \\
90 & $4.16(-4)$ & $2.51(-4)$ & $1.29(-4)$ \\
100 & $3.45(-4)$ & $2.46(-4)$ & $1.19(-4)$ \\
110 & $2.47(-4)$ & $2.42(-4)$ & $1.04(-4)$ \\
120 & $2.09(-4)$ & $2.47(-4)$ & $9.44(-5)$ \\
130 & $2.59(-4)$ & $2.73(-4)$ & $9.49(-5)$ \\
140 & $3.81(-4)$ & $3.18(-4)$ & $1.10(-4)$ \\
150 & $5.39(-4)$ & $3.67(-4)$ & $1.42(-4)$ \\
160 & $6.94(-4)$ & $3.96(-4)$ & $1.77(-4)$ \\
170 & $8.03(-4)$ & $3.98(-4)$ & $2.03(-4)$ \\
180 & $8.42(-4)$ & $3.93(-4)$ & $2.12(-4)$ \\
Total Dw & $2.99(-2)$ & $3.29(-2)$ & $2.98(-2)$ \\
Born & $3.80(-2)$ & $3.84(-2)$ & $3.45(-2)$ \\
\hline
\end{tabular}

Table 4. Calculated DW differential and integral cross sections (in $10^{-16} \mathrm{~cm}^{2} \mathrm{sr}^{-1}$ and $10^{-15} \mathrm{~cm}^{2}$ and Born integral cross sections for excitation of the ${ }^{1} \Delta_{\mathrm{u}}$ state.

\begin{tabular}{llll}
\hline & \multicolumn{3}{l}{ Energy $(\mathrm{eV})$} \\
\cline { 2 - 4 } Angle (deg) & 30 & 40 & 50 \\
\hline 10 & $7.18(-2)$ & $9.99(-2)$ & $1.17(-1)$ \\
20 & $6.92(-2)$ & $8.65(-2)$ & $9.33(-2)$ \\
30 & $6.04(-2)$ & $6.79(-2)$ & $6.92(-2)$ \\
40 & $5.06(-2)$ & $5.37(-2)$ & $5.61(-2)$ \\
50 & $4.42(-2)$ & $4.58(-2)$ & $4.98(-2)$ \\
60 & $4.40(-2)$ & $4.30(-2)$ & $4.36(-2)$ \\
70 & $5.03(-2)$ & $4.52(-2)$ & $3.84(-2)$ \\
80 & $6.11(-2)$ & $5.16(-2)$ & $3.71(-2)$ \\
90 & $7.27(-2)$ & $5.92(-2)$ & $3.94(-2)$ \\
100 & $8.11(-2)$ & $6.49(-2)$ & $4.17(-2)$ \\
110 & $8.29(-2)$ & $6.55(-2)$ & $4.20(-2)$ \\
120 & $7.73(-2)$ & $5.99(-2)$ & $4.01(-2)$ \\
130 & $6.63(-2)$ & $4.89(-2)$ & $3.52(-2)$ \\
140 & $5.36(-2)$ & $3.56(-2)$ & $2.70(-2)$ \\
150 & $4.21(-2)$ & $2.38(-2)$ & $1.95(-2)$ \\
160 & $3.34(-2)$ & $1.57(-2)$ & $1.76(-2)$ \\
170 & $2.79(-2)$ & $1.15(-2)$ & $2.08(-2)$ \\
180 & $2.60(-2)$ & $1.03(-2)$ & $2.32(-2)$ \\
Total Dw & $7.67(-1)$ & $6.57(-1)$ & $5.40(-1)$ \\
Born & $8.16(-3)$ & $6.52(-3)$ & $5.29(-3)$ \\
\hline
\end{tabular}


Table 5. Calculated $\mathrm{DW}$ differential and integral cross sections (in $10^{-16} \mathrm{~cm}^{2} \mathrm{sr}^{-1}$ and $10^{-16} \mathrm{~cm}^{2}$ ) for excitation of the ${ }^{3} \Sigma_{u}^{+}$state.

\begin{tabular}{llll}
\hline & \multicolumn{3}{c}{ Energy (eV) } \\
\cline { 2 - 4 } Angle (deg) & 30 & 40 & 50 \\
\hline 0 & $2.37(-3)$ & $2.01(-3)$ & $8.79(-4)$ \\
10 & $4.31(-3)$ & $3.52(-3)$ & $2.29(-3)$ \\
20 & $6.38(-3)$ & $4.34(-3)$ & $3.14(-3)$ \\
30 & $5.10(-3)$ & $2.41(-3)$ & $1.67(-3)$ \\
40 & $3.75(-3)$ & $1.73(-3)$ & $9.53(-4)$ \\
50 & $5.62(-3)$ & $3.45(-3)$ & $1.59(-3)$ \\
60 & $8.97(-3)$ & $5.15(-3)$ & $2.52(-3)$ \\
70 & $1.09(-2)$ & $5.99(-3)$ & $3.34(-3)$ \\
80 & $1.08(-2)$ & $6.11(-3)$ & $3.44(-3)$ \\
90 & $9.22(-3)$ & $5.21(-3)$ & $2.65(-3)$ \\
100 & $6.85(-3)$ & $3.70(-3)$ & $1.75(-3)$ \\
110 & $5.12(-3)$ & $2.84(-3)$ & $1.44(-3)$ \\
120 & $5.54(-3)$ & $3.21(-3)$ & $1.55(-3)$ \\
130 & $8.73(-3)$ & $4.31(-3)$ & $1.62(-3)$ \\
140 & $1.40(-2)$ & $5.49(-3)$ & $1.59(-3)$ \\
150 & $2.02(-2)$ & $6.54(-3)$ & $1.83(-3)$ \\
160 & $2.56(-2)$ & $7.48(-3)$ & $2.48(-3)$ \\
170 & $2.93(-2)$ & $8.20(-3)$ & $3.19(-3)$ \\
180 & $3.07(-2)$ & $8.47(-3)$ & $3.49(-3)$ \\
Total & $1.14(-1)$ & $5.59(-2)$ & $2.67(-2)$ \\
\hline
\end{tabular}

Table 6. Calculated DW differential and integral cross sections (in $10^{-16} \mathrm{~cm}^{2} \mathrm{sr}^{-1}$ and $10^{-16} \mathrm{~cm}^{2}$ ) for excitation of the ${ }^{3} \Pi_{u}$ state.

\begin{tabular}{llll}
\hline & \multicolumn{3}{c}{ Energy (eV) } \\
\cline { 2 - 4 } Angle (deg) & 30 & 40 & 50 \\
\hline 0 & $5.69(-3)$ & $5.03(-3)$ & $1.52(-3)$ \\
10 & $5.62(-3)$ & $4.39(-3)$ & $1.39(-3)$ \\
20 & $4.92(-3)$ & $2.83(-3)$ & $9.84(-4)$ \\
30 & $3.35(-3)$ & $1.44(-3)$ & $5.29(-4)$ \\
40 & $1.95(-3)$ & $9.40(-4)$ & $3.49(-4)$ \\
50 & $1.52(-3)$ & $9.28(-4)$ & $3.32(-4)$ \\
60 & $1.72(-3)$ & $8.65(-4)$ & $3.06(-4)$ \\
70 & $1.79(-3)$ & $6.92(-4)$ & $2.69(-4)$ \\
80 & $1.43(-3)$ & $5.04(-4)$ & $2.41(-4)$ \\
90 & $8.98(-4)$ & $3.45(-4)$ & $2.02(-4)$ \\
100 & $5.62(-4)$ & $2.62(-4)$ & $1.54(-4)$ \\
110 & $5.26(-4)$ & $2.79(-4)$ & $1.11(-4)$ \\
120 & $6.60(-4)$ & $3.47(-4)$ & $9.41(-5)$ \\
130 & $7.88(-4)$ & $4.00(-4)$ & $1.05(-4)$ \\
140 & $8.30(-4)$ & $4.37(-4)$ & $1.28(-4)$ \\
150 & $8.12(-4)$ & $4.94(-4)$ & $1.67(-4)$ \\
160 & $7.93(-4)$ & $5.57(-4)$ & $2.47(-4)$ \\
170 & $8.00(-4)$ & $5.92(-4)$ & $3.38(-4)$ \\
180 & $8.08(-4)$ & $5.99(-4)$ & $3.82(-4)$ \\
Total Dw & $1.72(-2)$ & $8.60(-3)$ & $3.24(-3)$ \\
\hline
\end{tabular}


Table 7. Calculated DW differential and integral cross sections (in $10^{-16} \mathrm{~cm}^{2} \mathrm{sr}^{-1}$ and $10^{-16} \mathrm{~cm}^{2}$ ) for excitation of the ${ }^{3} \Pi_{\mathrm{g}}$ state.

\begin{tabular}{llll}
\hline & \multicolumn{3}{c}{ Energy (eV) } \\
\cline { 2 - 4 } Angle (deg) & 30 & 40 & 50 \\
\hline 0 & $3.57(-3)$ & $1.36(-3)$ & $4.23(-4)$ \\
10 & $3.35(-3)$ & $1.82(-3)$ & $4.58(-4)$ \\
20 & $3.41(-3)$ & $2.69(-3)$ & $6.01(-4)$ \\
30 & $4.27(-3)$ & $2.93(-3)$ & $7.09(-4)$ \\
40 & $4.87(-3)$ & $2.41(-3)$ & $6.77(-4)$ \\
50 & $4.26(-3)$ & $1.86(-3)$ & $6.32(-4)$ \\
60 & $2.83(-3)$ & $1.65(-3)$ & $5.74(-4)$ \\
70 & $1.61(-3)$ & $1.51(-3)$ & $4.94(-4)$ \\
80 & $1.18(-3)$ & $1.39(-3)$ & $4.75(-4)$ \\
90 & $1.46(-3)$ & $1.46(-3)$ & $5.23(-4)$ \\
100 & $2.08(-3)$ & $1.71(-3)$ & $5.44(-4)$ \\
110 & $2.67(-3)$ & $1.90(-3)$ & $4.76(-4)$ \\
120 & $2.97(-3)$ & $1.88(-3)$ & $3.74(-4)$ \\
130 & $2.98(-3)$ & $1.76(-3)$ & $3.33(-4)$ \\
140 & $3.16(-3)$ & $1.88(-3)$ & $3.85(-4)$ \\
150 & $4.25(-3)$ & $2.42(-3)$ & $5.16(-4)$ \\
160 & $6.45(-3)$ & $3.17(-3)$ & $7.24(-4)$ \\
170 & $8.89(-3)$ & $3.77(-3)$ & $9.56(-4)$ \\
180 & $9.83(-3)$ & $3.98(-3)$ & $1.06(-3)$ \\
Total Dw & $3.67(-2)$ & $2.38(-2)$ & $6.48(-3)$ \\
\hline
\end{tabular}

Table 8. Calculated DW differential and integral cross sections (in $10^{-16} \mathrm{~cm}^{2} \mathrm{sr}^{-1}$ and $10^{-16} \mathrm{~cm}^{2}$ ) for excitation of the ${ }^{3} \Delta_{u}$ state.

\begin{tabular}{llll}
\hline & \multicolumn{3}{c}{ Energy (eV) } \\
\cline { 2 - 4 } Angle (deg) & 30 & 40 & 50 \\
\hline 0 & $2.56(-3)$ & $2.01(-3)$ & $1.18(-3)$ \\
10 & $2.61(-3)$ & $2.05(-3)$ & $1.20(-3)$ \\
20 & $2.73(-3)$ & $2.13(-3)$ & $1.22(-3)$ \\
30 & $2.81(-3)$ & $2.18(-3)$ & $1.11(-3)$ \\
40 & $2.72(-3)$ & $2.04(-3)$ & $7.86(-4)$ \\
50 & $2.45(-3)$ & $1.65(-3)$ & $4.80(-4)$ \\
60 & $2.27(-3)$ & $1.31(-3)$ & $4.70(-4)$ \\
70 & $2.57(-3)$ & $1.45(-3)$ & $6.29(-4)$ \\
80 & $3.36(-3)$ & $2.10(-3)$ & $8.08(-4)$ \\
90 & $4.19(-3)$ & $2.83(-3)$ & $1.09(-3)$ \\
100 & $4.67(-3)$ & $3.14(-3)$ & $1.41(-3)$ \\
110 & $4.81(-3)$ & $2.82(-3)$ & $1.47(-3)$ \\
120 & $4.72(-3)$ & $2.14(-3)$ & $1.19(-3)$ \\
130 & $4.49(-3)$ & $1.53(-3)$ & $8.25(-4)$ \\
140 & $4.42(-3)$ & $1.19(-3)$ & $6.15(-4)$ \\
150 & $5.12(-3)$ & $1.09(-3)$ & $6.62(-4)$ \\
160 & $6.89(-3)$ & $1.19(-3)$ & $9.45(-4)$ \\
170 & $8.97(-3)$ & $1.46(-3)$ & $1.30(-3)$ \\
180 & $9.91(-3)$ & $1.60(-3)$ & $1.47(-3)$ \\
Total Dw & $4.78(-2)$ & $2.50(-2)$ & $1.16(-2)$ \\
\hline
\end{tabular}



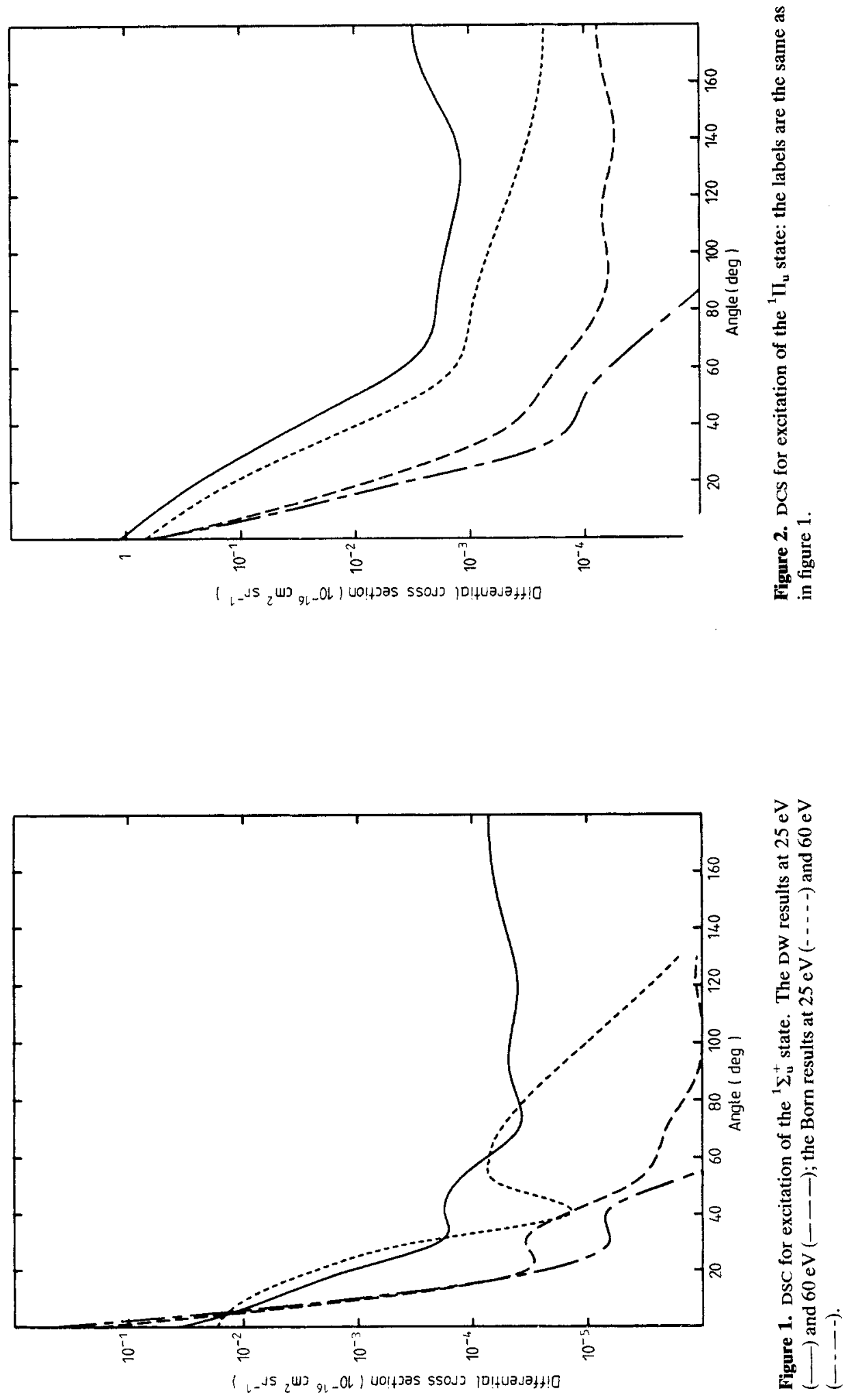

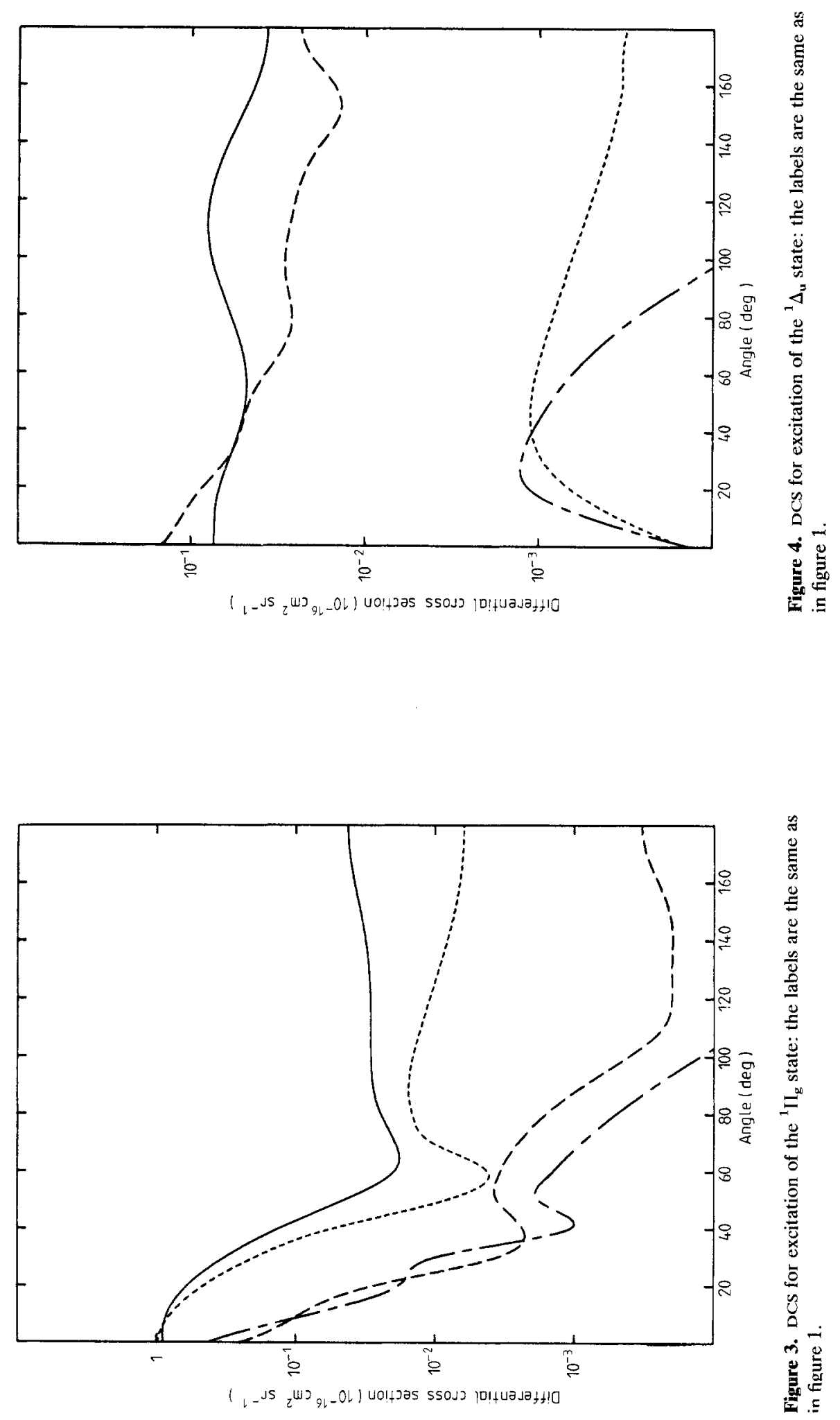

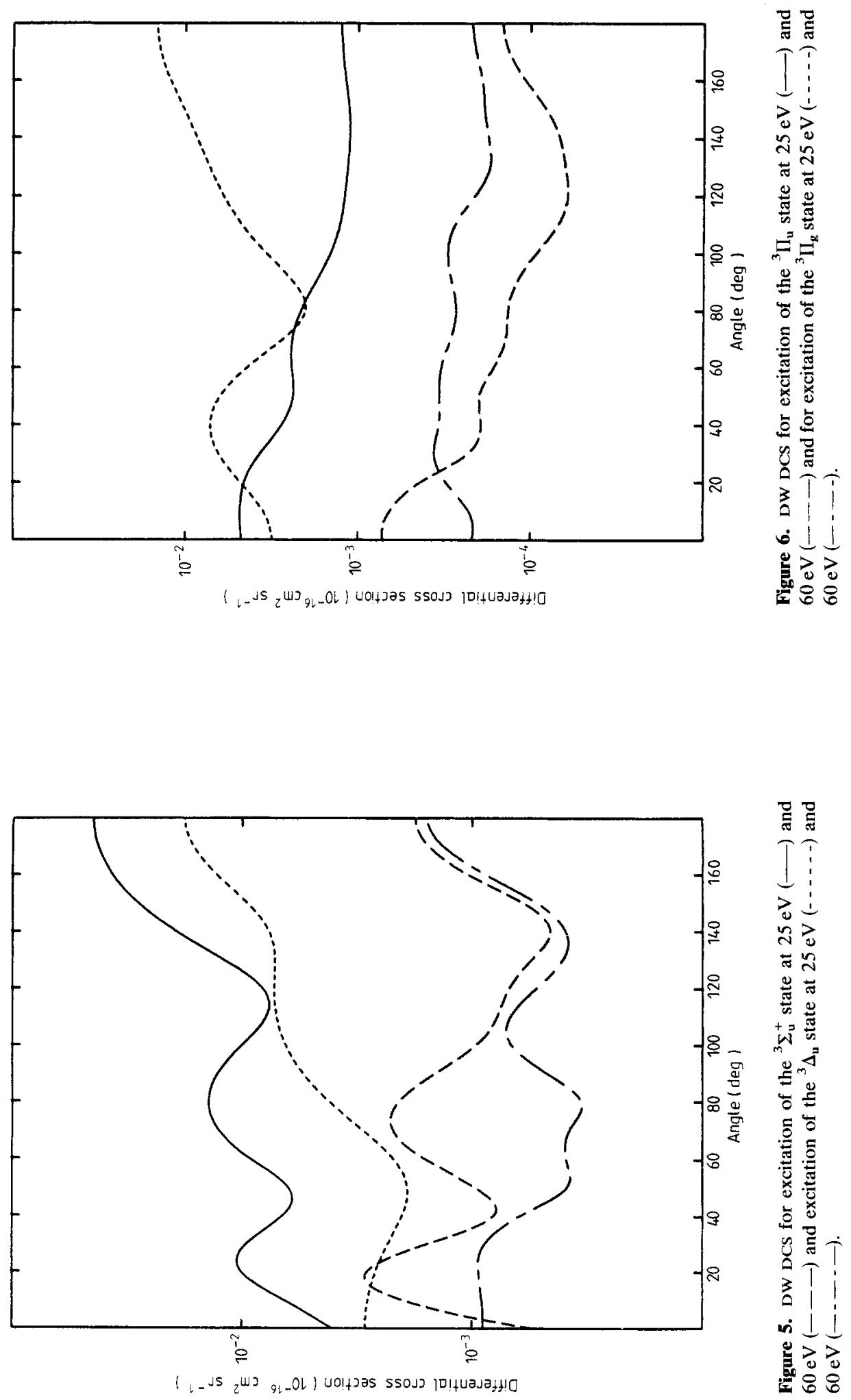
excitations we also show the Born DCs at the same energies. There are no experimental measurements with which to compare the present results. The qualitative behaviour of the DW DCS seems reasonable. For the dipole-allowed transitions $\left({ }^{1} \Sigma_{u}^{+}\right.$and ${ }^{1} \Pi_{u}$, figures 1 and 2 respectively) the DCS are strongly forward peaked, as expected, especially at higher impact energies. The DCs for excitation of the ${ }^{1} \Pi_{\mathrm{g}}$ state are also forward peaked in this energy range. For singlet-triplet excitations, the DCs are, in general, backward peaked at lower impact energies. The variation of the magnitude of DCS as a function of scattering angles is smaller than for the singlet-singlet cases.

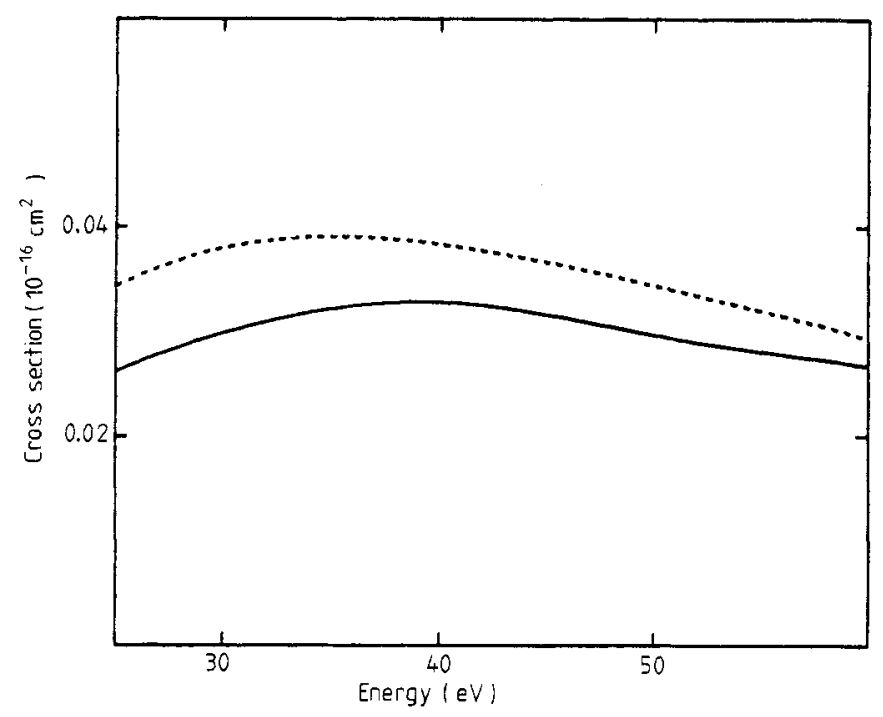

Figure 7. Total cross section for excitation of the ${ }^{1} \Sigma_{u}^{+}$state: DW results (full curve) and Born results (broken curve).

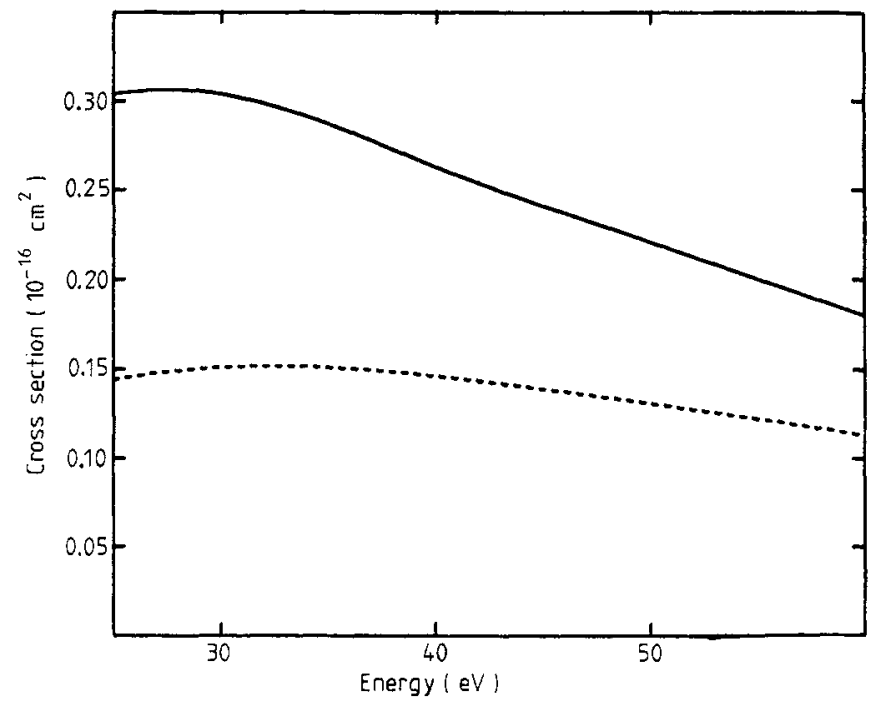

Figure 8. Total cross sections for excitation of the ${ }^{1} I_{u}$ state: the labels are the same as in figure 7. 
In figures 7 to 10 , we present the DW and Born total cross sections for excitation of the ${ }^{1} \Sigma_{\mathrm{u}}^{+},{ }^{1} \Pi_{\mathrm{u}},{ }^{1} \Pi_{\mathrm{g}},{ }^{1} \Pi_{\mathrm{g}}$ and ${ }^{1} \Delta_{\mathrm{u}}$ states respectively. We note that in the first three cases, the agreement between the DW and Born results is reasonable and becomes better at higher impact energies. However, for excitation of the ${ }^{1} \Delta_{u}$ state, the agreement between the DW and Born results is very poor even at $60 \mathrm{eV}$. This behaviour is unusual since at high impact energies the DW theory should approach the Born limit. The DW total cross sections for excitation of the triplet states are shown in figure 11. As expected, the total cross sections for excitation of these states diminish

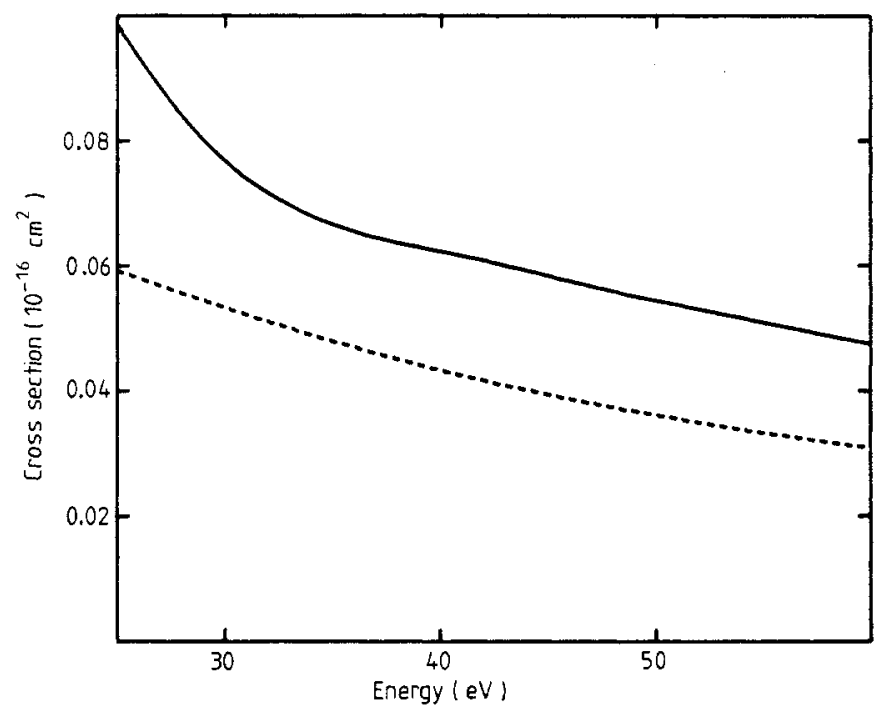

Figure 9. Total cross sections for excitation of the ${ }^{1} \Pi_{8}$ state: the labels are the same as in figure 7.

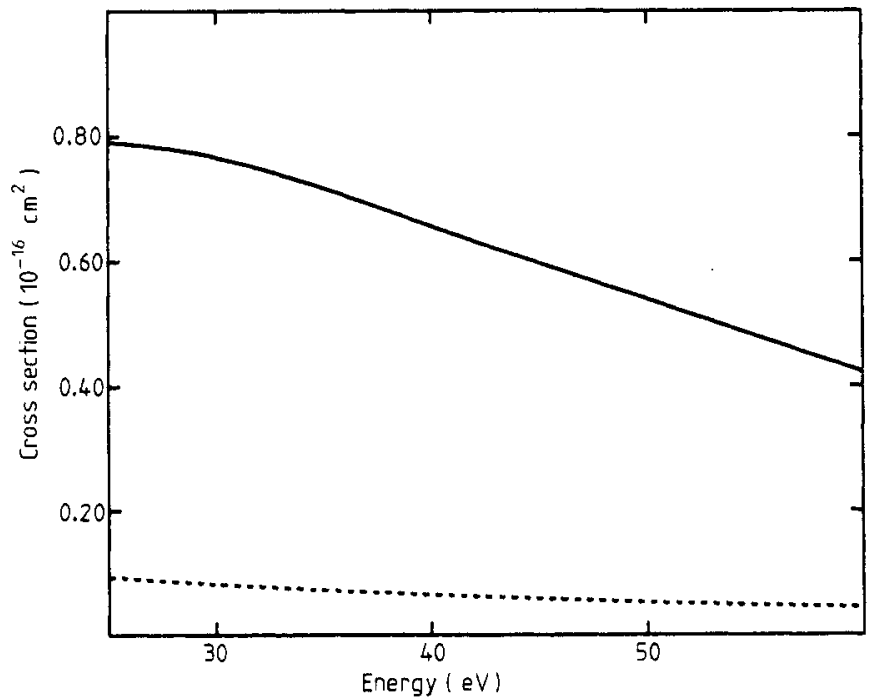

Figure 10. Total cross sections for excitation of the ${ }^{1} \Delta_{u}$ state: the DW results (full curve) and the Born results multiplied by ten (broken curve). 


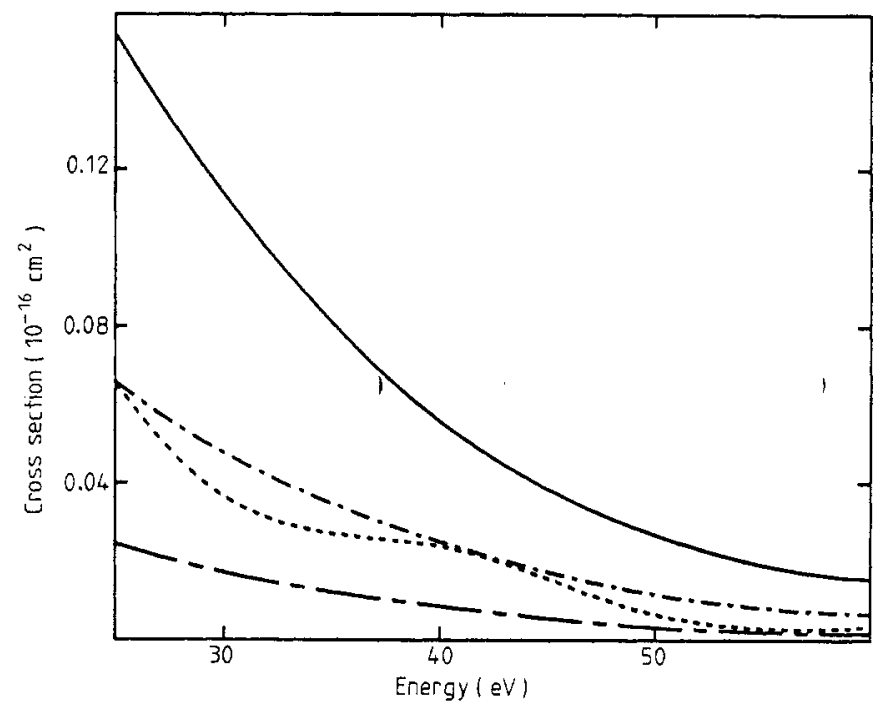

Figure 11. Total cross sections for excitation of the ${ }^{3} \Sigma_{u}^{+}(-),{ }^{3} \Delta_{u}(-\cdot-\cdot-),{ }^{3} \Pi_{g}$ (- . - - ) and ${ }^{3} \Pi_{u}(-\ldots-)$ states.

rapidly with increasing impact energies. Finally, previous applications of this method to $\mathrm{e}-\mathrm{N}_{2}$ collisions (Lee and McKoy 1982a) showed that the ${ }^{2} \Pi_{\mathrm{g}}$ shape resonance in the elastic scattering cross section leads to a spurious enhancement of the inelastic cross sections at outgoing channel energies equal to the resonance energy. The ${ }^{2} \Pi_{u}$ shape resonance would lead to a similar behaviour in e- $\mathrm{CO}_{2}$ collisions but this behaviour is not seen in the present results since the lowest incident energy here is $25 \mathrm{eV}$ and hence no outgoing channel energies lie close to the resonance energy of about $4 \mathrm{eV}$.

\section{Acknowledgments}

We thank Professor W A Goddard III for making some of his computer codes available to us. This research is based upon work supported by the National Science Foundation (Office of International Programs/US-Brasil) under Grant No INT80-01474. One of us (LMT) acknowledges the financial support of the CNPq (Brasil). The research reported in this paper made use of the CRAY-1 Computer of the National Center of Atmospheric Research (NCAR), Boulder, Colorado, which is supported by the National Science Foundation. Part of the calculations were done on the Dreyfus-NSF Theoretical Chemistry computer which is funded through grants from the Camille and Henry Dreyfus Foundation, the National Science Foundation (Grant No CHE7820233), and the Sloan Fund of the California Institute of Technology.

\section{References}

Dunning T H 1970 J. Chem. Phys. 53 2823-33

Fliffet A W and McKoy V 1980 Phys. Rev. A 21 1863-75 
Hall R I, Chutjian A and Trajmar S 1973 J. Phys. B: At. Mol. Phys. 6 1264-7

Hubin-Franskin M J and Collins J E 1971 Bull. Soc. R. Sci. Liege 40361

Inn E C Y, Watanabe K and Zelikof M 1953 J. Chem. Phys. 21 1648-50

Klump K N and Lassettre E N 1978 J. Electron Spectrosc. Relat. Phenom. 14 215-30

Land J E 1978 J. Appl. Phys. 49 5716-21

Lassettre EN and Shiloff J C 1965 J. Chem. Phys. 43 560-71

Lassettre E N, Skerbele A, Dillon M A and Ross K J 1968 J. Chem. Phys. 48 5066-96

Lee M T, Lucchese R R and McKoy V 1982 Phys. Rev. A 26 3240-8

Lee M T and McKoy V 1982a Phys. Rev. A in press

1982b J. Phys. B: At. Mol. Phys. 15 3971-83

Lucchese R R and McKoy V 1982 Phys. Rev. A 25 1963-8

Lucchese R R, Watson D and McKoy V 1980 Phys. Rev. A 22 421-6

McCurdy C W Jr and McKoy V 1974 J. Chem. Phys. $612820-6$

Meyer V D and Lassettre E N $1965 J$. Chem. Phys. 42 3436-41

Price W C and Simpson D M 1938 Proc. $R$. Soc. A 169 501-12

Rabalais J W, McDonald J M, Scherr V and McGlynn S P 1971 Chem. Rev. 71 73-108

Strickland D J and Green A E S 1969 J. Geophys. Res. 74 6415-24

Tanaka Y, Jursa A S and LeBlanc F J 1960 J. Chem. Phys. 32 1199-205 